\title{
The glucocerebrosidase locus in Gaucher's disease: molecular analysis of a lysosomal enzyme
}

\author{
P K Mistry, T M Cox
}

Since the concept of lysosomal storage disorders was introduced by Hers in $1965^{1}$ more than 30 inherited defects affecting this organelle have been recognised. ${ }^{2}$ Of these the most prevalent is the autosomal recessive condition Gaucher's disease, which is caused by deficiency of glucocerebrosidase (EC 3.2.1.45). Rapid progress has been made in understanding the molecular basis of Gaucher's disease and in the development of effective enzyme replacement therapy. Analysis of mutations that cause Gaucher's disease will shed light on structure-function relationships of the glucocerebrosidase molecule and should define the influence of genotype on disease expression. Molecular analysis of the glucocerebrosidase gene can assist the genetic counselling of families affected by Gaucher's disease and in the prognosis and treatment of individual patients with this condition.

\section{Historical perspective}

Gaucher's disease was first described by Phillippe Gaucher in 1882, who reported a patient with hepatosplenomegaly in whom the characteristic abnormal macrophages were thought to represent a primary neoplasm of the spleen. ${ }^{3}$ Identification of the stored material as glucocerebroside in 1934 was followed many years later by the finding that glucocerebrosidase activity was markedly reduced in the spleen of patients with Gaucher's disease by Brady et $a l^{4}$ and Patrick ${ }^{5}$ independently in 1965. Although the enzyme is deficient in most tissues of the body, it is the cellular specificity of the substrate that determines which tissues are principally affected. The substrate, glucocerebroside, is a constituent of complex glycolipids and is released in the breakdown of cell membranes, particularly leucocytes and erythrocytes, by mononuclear phagocytes. Thus, in Gaucher's disease engorged macrophages accumulate throughout the body and cause hepatosplenomegaly, bone marrow infiltration with skeletal disease, and, rarely, involvement of the lungs and brain.

\section{Classification of Gaucher's disease}

There are three main clinical subtypes of Gaucher's disease. ${ }^{6}$ Most patients suffer from visceral involvement in the absence of neurological disease: this non-neuronopathic form of the condition is known as type I Gaucher's disease. Within this group there is great variability in the rate of disease progression. Patients may present in childhood with hepatosplenomegaly, pancytopenia, bone necrosis, and osteoporosis or come to light in the ninth decade of life because of the incidental finding of splenomegaly. Type I Gaucher's disease occurs rarely in all ethnic groups but it is more frequent in the Ashkenazi Jewish population. A wide range of disease activity is seen in the Jewish population but patients with late onset or mild disease are usually found to be of Ashkenazi descent. ${ }^{6}$ Type II (acute neuronopathic) disease is a rare disorder with no ethnic predilection. It causes a rapidly progressive neurovisceral storage disease and death in infancy. Affected infants come to light as a result of oculomotor abnormalities, cranial and bulbar nerve palsies, spasticity, convulsions, and opisthotonus. Type III (subacute neuronopathic) Gaucher's disease progresses less rapidly but is associated with ataxia, myoclonus, and convulsions; there is progressive intellectual impairment which is accompanied by hepatosplenomegaly and skeletal disease. Death usually occurs in childhood or adolescence. This type of Gaucher's disease is also rare but it is frequent in a genetic isolate that represents a single extended pedigree originating from the Norrbottnian region of northern Sweden. ${ }^{7}$

\section{Diagnosis}

Typically the diagnosis of Gaucher's disease is established by the finding of characteristic glycolipid laden large macrophages on histological examination of bone marrow, the liver, or spleen. ${ }^{68}$ These findings are not absolutely specific since pseudo-Gaucher's cells may appear in the marrow of patients suffering from a variety of conditions associated with increased cell turnover, including chronic myeloid leukaemia and thalassaemia. ${ }^{9}$ Determination of leucocyte acid $\beta$-glucosidase activity remains a reliable and simple means of confirming the diagnosis of Gaucher's disease. ${ }^{610}$

Deficiency of glucocerebrosidase results from defects in the enzyme protein itself but a
Correspondence to Dr Mistry. 
few patients have been reported in whom a deficiency of a heat stable activator (SAP2sphingolipid activator protein 2) of glucocerebrosidase is responsible for a phenocopy of Gaucher's disease. ${ }^{11}$ Biochemical studies of the residual glucocerebrosidase activity in patients with Gaucher's disease, combined with the wide spectrum of clinical presentation, had led to the expectation that multiple mutant alleles would be responsible for the condition. ${ }^{12}$ Landmarks in the molecular analysis of Gaucher's disease include the purification of glucocerebrosidase from human placenta in $1973,{ }^{13}$ the cloning of glucocerebrosidase cDNA by Sorge et al $^{14}$ in 1985, and the complete sequence characterisation of the human glucocerebrosidase gene and its pseudogene by Horowitz et al ${ }^{15}$ in 1989.

\section{Glucocerebrosidase gene and pseudogene}

The structural gene for glucocerebrosidase and its pseudogene map to human chromosome $1 \mathrm{q} 21 . .^{15}$ The structural gene contains 11 exons and spans 7.6 kilobases; the pseudogene is located $16 \mathrm{~kb}$ downstream. In the homologous regions, $96 \%$ nucleotide identity exists. Promoter regions of genes encoding lysosomal enzymes usually conform to the housekeeping model. The promoter of the glucocerebrosidase functional gene is unusual in this respect since it contains TATA and CAAT boxes but no Sp1 binding site. ${ }^{15} \mathrm{~A}$ promoter of the pseudogene can direct transcription of reporter genes and pseudogene transcripts have been isolated from extracts of normal cells maintained in culture. ${ }^{1617}$ These transcripts, however, cannot be translated since they contain multiple stop codons; in addition, exons are deleted during mRNA processing because of mutations in consensus splice sites. The pseudogene is smaller than the structural gene because of sequence loss in introns 2 ( $313 \mathrm{bp}$ ), 4 (626 bp), 6 (320 bp), and 7 (277 bp) as well as two missing exon sequences in exon $9(55 \mathrm{bp})$ and exon $4(5 \mathrm{bp})$. The missing intron sequences appear to be $A l u$ sequences that have been inserted into the functional gene after the presumed duplication event that gave rise to the pseudogene. ${ }^{15}$ In addition, the pseudogene contains multiple point substitutions throughout its sequence: when transferred to the structural gene by gene conversion or recombination events, these mutations result in defects in glucocerebrosidase that cause Gaucher's disease. Such homologies and differences between the functional and pseudogene have important implications for investigation of mutations in Gaucher's disease and, as a result of transcription of the pseudogene, particularly affect the molecular analysis of glucocerebrosidase cDNA.

Complementary DNA encoding human glucocerebrosidase spans about $2 \mathrm{~kb}$. The mRNA is unusual in that it has two functional AUG start codons ( -57 and -118 nucleotides) but translation initiated at either site results in functional glucocerebrosidase. ${ }^{1418}$ Northern analysis indicates the presence of three gluco- cerebrosidase mRNA species of approximately $5 \cdot 6,2 \cdot 5$, and $2 \cdot 0 \mathrm{~kb}$. The longest mRNA species is thought to represent an incompletely spliced nuclear transcript whereas the shorter transcripts appear to arise from alternative sites for transcriptional initiation and polyadenylation. ${ }^{19}$ As expected for a lysosomal enzyme, a 19 amino acid hydrophobic signal sequence is present. The mRNA is translated into a protein that is cleaved to produce a mature polypeptide of 497 amino acids with a molecular weight of $55.5 \mathrm{kDa}$. The polypeptide contains five potential 0 linked glycosylation sites, four of which appear to be glycosylated. The active site of glucocerebrosidase resides in the C-terminal half of the molecule and investigations with covalently bound inhibitors suggest a critical role for asp ${ }^{443}$ and asp ${ }^{358}$ in catalysis. ${ }^{20}$

Numerous polymorphic sites have been identified in the glucocerebrosidase gene, eight in introns and three in the $5^{\prime}$ untranslated region. ${ }^{21}$ These sequences are in linkage disequilibrium and constitute two main haplotypes. Various mechanisms have been proposed to explain the ascendancy of these haplotypes. A search for further polymorphic sites might facilitate detection of carriers in relatives of patients in whom the nature of the mutation has not been established. Previous studies have identified a common polymorphism with respect to a PvuII restriction site at genomic nucleotide position 3931 in intron 6 which has been linked to many uncharacterised alleles in patients with Gaucher's disease. $^{22}$

\section{Mutational analysis in Gaucher's disease} HETEROGENEITY OF MUTATIONS

The multiplicity of mutations responsible for Gaucher's disease had long been suspected from the clinical heterogeneity of the condition and the different kinetic properties of the residual enzyme activity. ${ }^{12}$ Marked differences in the synchesis and processing of enzyme protein had also been shown, ${ }^{18}$ but the extent of the heterogeneity of lesions at the glucocerebrosidase locus has only recently become evident as a result of detailed molecular analysis. Excellent reviews, to which the reader is referred, catalogue these mutations. ${ }^{182324}$ In Ashkenazi Jews with type I disease, four mutations account for over $98 \%$ of disease alleles. These same four mutations account for approximately $60 \%$ of disease alleles in patients not known to have Jewish ancestry; rare or private mutations account for the remainder. ${ }^{25}{ }^{26}$ Many patients with Gaucher's disease exhibit compound heterozygosity for mutations at the glucocerebrosidase locus.

\section{COMMON MUTATIONS}

Molecular lesions in the glucocerebrosidase gene described hitherto include missense mutations, a splice site mutation, a nucleotide insertion, deletions, crossovers between the structural gene and pseudogene, and gene conversion events (non-homologous recombi- 
nation). The most prevalent mutation in nonneuronopathic Gaucher's disease is asn ${ }^{370} \rightarrow$ ser (N370S) which is caused by replacement of an A by $\mathrm{G}$ at cDNA nucleotide position $1226 .{ }^{27}$ This mutation accounts for $75 \%$ of disease alleles among Ashkenazi patients and approximately $25 \%$ among patients not known to be Jewish. ${ }^{25}$ In vitro expression of this mutant enzyme in insect cells indicates that, compared with the wild type enzyme, it has reduced specific activity and other abnormal catalytic properties in relation to activation by negatively charged phospholipids and SAP2. ${ }^{29}$ In Ashkenazi Jews the N370S mutation is linked to a PvuII polymorphism; it is invariably found in the context of the Pv1.1- genotype. $^{22}$ The observation that most unknown alleles in Ashkenazi patients occurred in the context of the Pvl.1 $1^{+}$genotype prompted Beutler et $a l^{30}$ to search for other widespread mutations in this ethnic group. Recently they reported the insertion of a single nucleotide, $G$, at cDNA position 84 , that results in a frameshift that abolishes translation of glucocerebrosidase. ${ }^{30}$ This mutation has so far only been found as a single copy in compound heterozygotes of Ashkenazi descent and accounts for $13 \%$ of disease alleles. A less common missense mutation, leu ${ }^{444} \rightarrow$ pro (L444P) is caused by replacement of a $\mathrm{T}$ for a $\mathrm{C}$ at $\mathrm{cDNA}$ nucleotide $1448 .^{31}$ This mutation occurs normally in the pseudogene and, thus, has the potential to complicate molecular analysis of the structural gene in Gaucher's disease. ${ }^{15}$ The L444P mutation accounts for only about $2 \%$ of disease alleles in Ashkenazi patients but for approximately $40 \%$ of the alleles in non-Jewish patients. ${ }^{25} 32$ The mutation is associated with all three subtypes of disease but tends to cause severe or neuronopathic disease when present in the homozygous form. Leucine 444 is located in the catalytic domain previously identified in the enzyme by reaction with the substrate analogue $\beta$-conduritol epoxide. ${ }^{20}$ Disruption of the protein structure in this region produces an unstable protein that possesses little residual activity. ${ }^{33}$

Lately, Beutler et $a l^{34}$ have described a splicing mutation (IVS2 + 1) that results in the deletion of exon 2 from the mature transcript. This accounts for approximately $3 \%$ of disease mutations in unrelated Jewish patients. ${ }^{32}$ The IVS2 +1 mutation (replacement of $\mathrm{G}$ by $\mathrm{A}$ at genomic position 1067) is also normally present in the glucocerebrosidase pseudogene. Several mutant alleles of glucocerebrosidase differ from the wild type sequence in as many as four codons and correspond to sequence variations found in the pseudogene. These complex alleles represent chimeric molecules, part functional gene and part pseudogene, that may result from gene conversion events or unequal crossing over. ${ }^{35-37}$

The most widespread missense mutations responsible for Gaucher's disease N370S, L444P, and R463C (cDNA 1226G, 1448C and $1504 \mathrm{~T}$ respectively) occur in residues at exons 9 and 10 which have been considered critical for the formation of the active site. However, other missense mutations occur throughout the gene (four in exon 5, two in exon 6, one in exon 7 , five in exon 8 , two in exon 11$)^{182432}$ and our understanding of the part played by these residues in the functional integrity of human glucocerebrosidase will depend in the first instance on the determination of the three dimensional structure of the enzyme at atomic resolution.

\section{Mutational analysis in clinical practice}

The human glucocerebrosidase gene has been subject to intensive study in relation to Gaucher's disease. Given the prevalence of Gaucher's disease and the morbidity associated with its more severe forms, there is a need for methods to facilitate prenatal screening and diagnosis. The emergence of an effective alternative to marrow transplantation in the treatment for non-neuronopathic Gaucher's disease also has a bearing on diagnosis and prognosis. Although enzymatic assay of acid $\beta$ glucosidase activity may serve to confirm the diagnosis of Gaucher's disease when suspected, activities obtained in up to $20 \%$ of obligate heterozygotes are within the normal range of enzymatic activity. Under these circumstances, molecular analysis of the glucocerebrosidase gene should aid the definitive detection of carriers in families at risk for Gaucher's disease. In addition, procedures based on the polymerase chain reaction for the analysis of genomic DNA should improve the potential for early prenatal diagnosis by chorionic villus sampling where there has been experience of neuronopathic Gaucher's disease (figure).

Molecular analysis of the glucocerebrosidase gene is complicated by the presence of the transcribed pseudogene and thus methods to detect mutations must either amplify the structural gene sequences selectively or allow separation of the amplified structural gene from the amplified pseudogene sequences. ${ }^{25} 38$ Screening for known mutations has relied on hybridisation to allele specific oligonucleotide probes or, more simply, when the mutation alters a restriction site, by digestion with appropriate restriction endonucleases. Examples of the latter include the $N c i$ I site associated with the L444P mutation, ${ }^{31}$ the HhaI site associated with the $\mathrm{P} 415 \mathrm{~N}$ mutation, ${ }^{39}$ and deletion of a $C f r 10$ site in association with N463C. ${ }^{25}$ However, most of the mutations that cause Gaucher's disease do not alter restriction sites and screening for these has been facilitated by the application of the 'mismatch PCR' technique. ${ }^{40}$ The search for known mutations, regardless of whether or not a restriction site is present, has been further simplified by the use of the amplification refractory mutation system (ARMS) which permits rapid genotyping based on allele specific amplification in the PCR in the single step ${ }^{25}$ (figure). These procedures allow genetic diagnosis of Gaucher's disease to be carried out in diagnostic laboratories outside major research centres. In our laboratory, among seven Ashkenazi Jewish patients with Gaucher's disease, 11 disease alleles were identified as N370S, and two as 


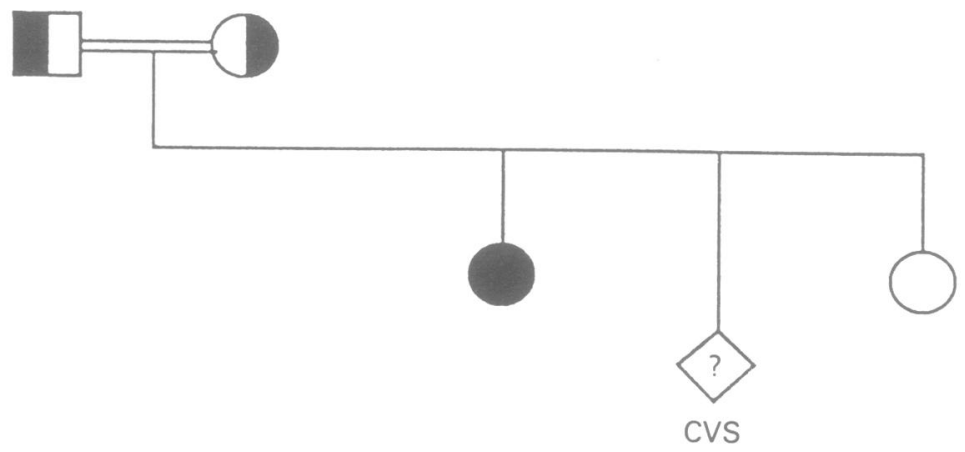

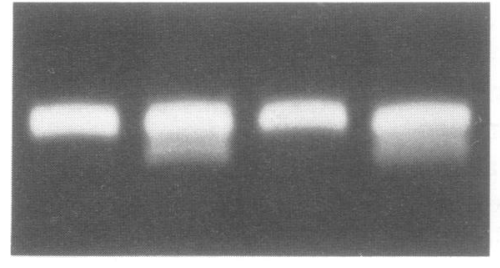

a
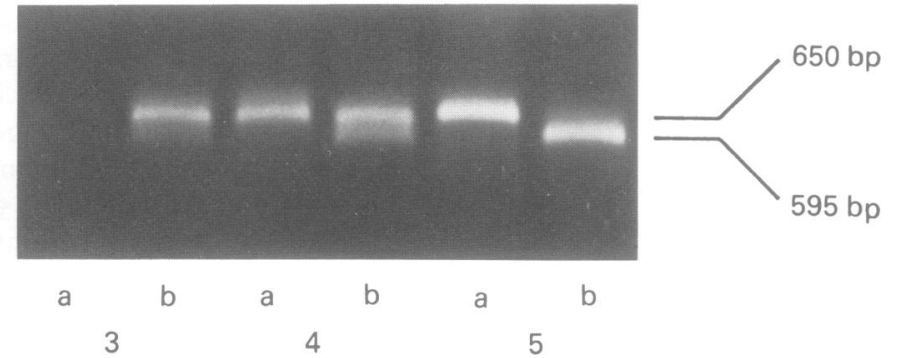

Prenatal diagnosis of the L444P mutation in the human glucocerebrosidase gene by ARMS analysis. The PCR was carried out simultaneously with a primer complementary to the wild type sequence ( $a$ ) and the mutant sequence (b). The pseudogene, which normally carries the L444P sequence, gives rise to a $55 \mathrm{bp}$ smaller product. 1 and 2 parents (first cousins); 3 affected child previously treated by bone marrow transplantation at the age of 2 years; 4 CVS: chorionic villous DNA obtained from a further pregnancy at 9 weeks' gestation. 5 DNA obtained from healthy daughter. The assignment of genotypes, including heterozygosity in the fetus, was later confirmed by digestion with the restriction endonuclease NciI.

84GG, leaving only one unknown allele. Among our other 17 patients from other ethnic backgrounds, ARMS analysis for common mutations showed six N370S alleles, nine L444P alleles, two N463C alleles, and one IVS $2+1$ leaving less than half the disease alleles in this diverse group of patients unidentified.

\section{Population genetics}

Gaucher's disease appears to be most common in Ashkenazi Jews in whom a recent survey has suggested that the frequency of homozygosity for mutations may be as high as 1 in $976 .{ }^{41}$ This is largely because of the prevalence of the N370S mutation which accounts for $75 \%$ of disease alleles. ${ }^{28}$ Other common mutations in this population are $84 \mathrm{GG}(13 \%)$, IVS $2+1$ $(3 \%)$, leaving less than $5 \%$ of alleles unknown..$^{30}$ As a result of studies in more than 2000 Ashkenazi subjects, Beutler et $a l^{41}$ estimated the gene frequency for N370S and the $84 \mathrm{GG}$ mutation to be 0.032 and 0.00217 , respectively; thus 1 in 980 offspring of unselected marriages between Ashkenazi Jews are predicted to be N370S homozygotes. In their survey, Beutler et al discovered four asymptomatic persons harbouring two copies of the N370S mutation when they examined a random Ashkenazi sample. The N370S mutation was found to be more prevalent than expected in the healthy Ashkenazi population in relation to a large sample of Ashkenazi patients with Gaucher's disease. Beutler et al estimated that approximately two-thirds of the alleles were missing from the patient population with Gaucher's disease, once again indicating variable expressivity of the homozygous form of this mutation and its association with relatively mild disease. This mutation exists at polymor- phic frequency among Ashkenazi Jews and raises the possibility that it confers a selective advantage in the heterozygous state. The N370S and 84GG mutations are found consistently in the context of their respective haplotype associations, an observation that suggests that they arose on a single ancestral chromosome and were selected for or spread by diffusion from a single founding population. The Swedish isolate found in Norrbotten is strongly associated with type III Gaucher's disease. ${ }^{7}$ All 10 patients studied were found to be homozygous for L444P and careful pedigree analysis later showed that these subjects were descended from a common ancestor in northern Sweden. ${ }^{72}$ Among non-Jewish patients, L444P is the most common disease allele and accounts for approximately $40 \%$ of disease alleles. In the general population, L444P occurs in the context of different haplotypes, a finding that suggests that this pathological variant has arisen repeatedly as a result of distinct mutational events. ${ }^{23}$

\section{Genotype-phenotype correlations in Gaucher's disease}

Many inherited diseases show variable clinical expressivity and Gaucher's disease is no exception: type II (neuronopathic disease) is a condition of infancy that progresses rapidly to death whereas other persons homozygous for other mutations in the glucocerebrosidase gene may remain asymptomatic in their ninth decade of life. Striking heterogeneity of clinical expression may also be observed in full sibs within a given pedigree affected by Gaucher's disease and provides clear evidence that factors outside the glucocerebrosidase locus may also influence the degree to which the enzymatic deficiency is responsible for clinical manifesta- 
tions. In general, it may be said that the more severe the disease the lower the level of residual enzymatic activity that can be expected.

The level of activity needed to protect against the more severe manifestations of Gaucher's disease may be a small fraction of the normal. Conzelman and Sandhoff ${ }^{43}$ proposed that there exists a critical threshold in the activity of lysosomal enzymes below which accumulation of undegraded substrate occurs. This critical threshold and the rate of accumulation of undegraded substrate may differ between cells, that is, between Kupffer cells and splenic macrophages or between the same cells at different developmental stages in different tissues, depending on the rate of influx of substrate. Thus a very small difference of residual activity could markedly affect the age of onset of the disease, the rate of its progression, and its clinical manifestations. An additional factor in relation to Gaucher's disease is that, during its course, when the spleen reaches a critical size, hypersplenism may result in the dramatic increase in glycolipid turnover from blood cell membranes. This would be expected to accelerate the deposition of undegraded substrate. Thus secondary pathophysiological disturbances may influence the apparent severity of Gaucher's disease disproportionately and lead to bias in the correlation of given genotypes with clinical manifestations. Other mechanisms may influence the activity of glucocerebrosidase in Gaucher's cells: there may be enhanced transcription, resulting in a 2 to 3 fold increase in steady state mRNA that would partially compensate for decreased enzymatic activity ${ }^{19}$ and partial amelioration of the disease.

Because of the implications for prognosis and counselling of carriers, an important objective in the molecular analysis of the glucocerebrosidase gene has been to correlate the nature of the mutation with the clinical features of the disease. As indicated above, precise correlation has been difficult to achieve in Gaucher's disease. Nevertheless, it is clear that genotype does influence phenotype strongly. Indices of disease severity, that is, age of presentation or a composite score based on other clinical criteria, have been used to estimate rates of disease progression. ${ }^{44}$ In general, homozygosity for the N370S mutation confers the mildest form of disease and, although the presence of another allele is usually associated with more aggressive features, expression of one copy of this mutated gene may indeed provide sufficient enzymatic activity to preclude development of neurological disease. Some homozygotes for N370S remain asymptomatic and have very few manifestations of the disease even on careful clinical study and, as indicated above, up to two-thirds of persons with this genotype may escape medical attention for Gaucher's disease. ${ }^{41}$ Among those who present with symptoms, the median age of detection is 30 years but a few subjects have a disease with onset in adolescence or early adult life. In one such case the presence of a deleted allele resulting in incorrect assignment of a homozygous genotype was later shown. ${ }^{24}$ Despite these exceptions there are clearly genetic and environmental factors that influence the severity of the disease but which are not yet understood. The role of variation in the expression or quality of SAP 2 in relation to disease expression has yet to be systematically investigated.

Homozygosity for the L444P mutation causes severe Gaucher's disease and correlates with neurological complications. Here again, however, striking variability in phenotype has been observed ranging from fulminant neurological involvement characteristic of type II disease to the more subacute neuronopathic form in type III. A few patients with the type I phenotype have been found to be homozygous for the L444P mutation. ${ }^{45}$ In some type II patients who were originally designated L444P homozygotes, numerous additional mutations in the glucocerebrosidase gene resulting from crossover events with the pseudogene have been found. ${ }^{35-37}$ The correlation with neurological complications of Gaucher's disease does not appear to apply in all population groups, however. In Japanese patients homozygosity for L444P has been found among the non-neuronopathic forms of Gaucher's disease and a different mutation has primarily been correlated with type III disease $\left(\mathrm{phe}^{213} \rightarrow \mathrm{ile}\right.$ ) (F213I). ${ }^{4647}$ Recent investigations of the effect of the R463C mutation illustrate another aspect of genotype-phenotype correlations in Gaucher's disease. In two studies R463C has been found to be associated with severe disease and neurological involvement, ${ }^{2545}$ but in another investigation it is associated with mild disease, ${ }^{23}$ yet when this mutant enzyme is expressed in insect cells, its specific activity has been found to be little impaired. However, the enzyme is also much less susceptible to activation by the sphingolipid activator protein in vitro. ${ }^{48}$

Thus, in any individual patient it is difficult to predict accurately the outcome of disease solely from knowledge of the genotype. To determine the origin of phenotypic variation is a scientific question of importance which may eventually lead to therapeutic approaches to disease modification, for example, in relation to the sphingolipid activator protein. The investigation of the source of phenotypic variation will be greatly helped by the development of methods to measure residual glucocerebrosidase activity in situ and this might be achieved by loading lysosomes in macrophages obtained from patients with Gaucher's disease with radiolabelled glucocerebroside or a suitable fluorescent artificial substrate. ${ }^{49}$ This would in the first place allow the hypothesis of Conzelman and Sandhoff ${ }^{43}$ to be examined. In the same way, the production of models of Gaucher's disease in experimental animals ${ }^{50}$ may also assist in the investigation of the cellular pathophysiology of glucocerebrosidase deficiency in man.

We wish to thank Drs Ian Ellis and Anthony Fensom of the Paediatric Research Unit, UMDS-Guy's Campus, London SE1 9RT for referring the family depicted in the figure 
for prenatal diagnosis and the Wellcome Trust for support. Mrs Joan Grantham kindly rendered the manuscript suitable for publication.

1 Hers HG. Inborn lysosomal storage diseases. Gastroenterology 1965;48:625-33.

Scriver CR, Beaudet AL, Sly WS, Valle D, eds. The metabolic basis of inherited diseases. 6th ed. New York: McGraw-Hill, 1989:1565-839.

3 Desnick RJ. Gaucher disease: a century of delineation and understanding. In: Desnick RJ, Gatt S, Grabowski GA eds. Gaucher disease: a century of delineation. New York Alan R Liss, 1982:1-33.

4 Brady RO, Kanfer JN, Shapiro D. Metabolism of glucocerebrosides. II. Evidence of an enzymatic deficiency in Gaucher's disease. Biochem Biophys Res Commun 1965;18:221-5.

5 Patrick AD. A deficiency of glucocerebrosidase in Gaucher's disease. Biochem f 1965;97:17C-18C

6 Barranger JA, Ginns EI. Glucosylceramide lipidosis: Gaucher disease. In: Scriver CR, Beaudet AL, Sly WS Valle $\mathrm{D}$, eds. The metabolic basis of inherited disease. 6th ed. New York: McGraw-Hill, 1989:1677-98.

7 Svennerholm L, Dreborg S, Erikson A, et al. Gaucher disease of Norrbottnian type (type 3 phenotypic manifestations). In: Desnick RJ, Gatt S, Grabowski GA, eds. Gaucher disease: a century of delineation. New York: Alan $R$ Liss, 1982:67-95.

8 Burns GF, Cowley JC, Flemans RJ, et al. Surface marker and other characteristics of Gaucher's cells. $\mathcal{f}$ Clin Pathol 1977;30:981-7.

9 Beutler E. Gaucher disease. Blood Rev 1988;2:59-70.

10 Beutler E, Kuhl WL. The diagnosis of the adult type Gaucher disease and its carrier state by demonstration of deficiency of $\beta$-glucosidase activity in peripheral blood deficiency of $\beta$-glucosidase activity in periphe
leucocytes. $f \mathrm{Lab}$ Clin Med 1970;76:747-55.

11 O'Brien JS, Kishimoto Y. Saposin proteins: structure, function and role in lysosomal storage disorders. FASEB f 1991;5:301-8.

12 Grabowski GA, Goldblatt J, Dinur T, et al. Genetic heterogeneity in Gaucher's disease and immunologic studies of residual enzyme in cultured fibroblasts from non-neurologic and neurologic patients. Am $\mathcal{f}$ Med Genet 1985;21:529-49.

13 Pentchev PG, Brady RO, Hibbert SR, Gal AE, Shapiro D. Isolation and characterization of glucocerebrosidase from human placental tissue. $\mathcal{F}$ Biol Chem 1973;248:5256.

14 Sorge J, West C, Westwood B. Beutler E. Molecular cloning and nucleotide sequence of human glucocerebro-

15 Horowitz M, Wilder S, Horowitz Z, Reiner O, Gelbart T Beutler $\mathrm{E}$. The human glucocerebrosidase gene and pseuBeutler E. The human glucocerebrosidase gene and pseu-
dogene: structure and evolution. Genomics 1989;4:87-96.

6 Reiner O, Wigderson M, Horowitz M. Structural analysis of human glucocerebrosidase genes. DNA 1988;7:107-16.

17 Sorge J, Gross E, West C, Beutler E. High level transcription of glucocerebrosidase pseudogene in normal subjects and patients with Gaucher's disease. $f$ Clin Invest 1990;86:1137-41.

18 Grabowski GA, Gatt S, Horowitz M. Acid $\beta$-glucosidase: enzymology and molecular biology of Gaucher disease. Crit Rev Biochem Mol Biol 1990;25:385-414.

19 Reiner O, Wilder S, Girol D, Horowitz M. Efficient in vitro and in vivo expression of human glucocerebrosidase cDNA. DNA 1987;6:101-8.

20 Dinur T, Osiecki KM, Legler G, Gatt S, Desnick RJ, Grabowski GA. Human acid $\beta$-glucosidase: isolation and amino acid sequence of a peptide containing the catalytic amino acid sequence of a peptide containing the
site. Proc Natl Acad Sci USA 1986;83:1660-4.

21 Beutler E, West C, Gelbart T. Polymorphisms in the human glucocerebrosidase gene. Genomics 1992;12:795800 .

22 Zimran A, Gelbart T, Beutler E. Linkage of the PvuII polymorphism with the common Jewish mutation for Gaucher disease. Am ₹ Hum Genet 1990;46:902-5.

23 Beutler E. Gaucher disease: new molecular approaches to diagnosis and treatment. Science 1992;256:794-9.

24 Beutler E, Gelbart T, West C. Identification of six new Gaucher disease mutations. Genomics 1993;15:203-5.

25 Mistry PK, Smith SJ, Ali M, Hatton CSR, McIntyre N, Cox TM. Genetic diagnosis of Gaucher's disease. Lance 1992;339:889-92.

26 Walley AJ, Barth ML, Ellis I, Fensom AH, Harris A Gaucher's disease in the United Kingdom: screening
non-Jewish patients for the two common mutations. 7 Med Genet 1993;30:280-3.

27 Tsuji S, Martin BM, Barranger JA, Stubblefield BK, La Marca ME, Ginns EI. Genetics heterogeneity in type I Gaucher disease: multiple genotypes in Ashkenazic and non-Ashkenazic individuals. Proc Natl Acad Sci USA non-Ashkenazic ind

28 Zimran A, Gelbart T, Westwood B, Grabowski GA, Beutler E. High frequency of the Gaucher disease mutation at nt 1226 among Ashkenazi Jews. Am $\mathcal{F}$ Hum Genet 1991;49:855-9.

29 Grace ME, Graves PN, Smith FI, Grabowski GA. Analysis of catalytic activity and inhibitor binding of human $\beta$ glucosidase by site-directed mutagenesis. $7 \mathrm{Biol} \mathrm{Chem}$ 1990;265:6827-35.

30 Beutler E, Gelbart T, Kuhl W, Sorge J, West C. Identification of the second common Jewish Gaucher disease mutation makes possible population-based screening for the heterozygote state. Proc Natl Acad Sci USA heterozygote sta.

31 Tsuji S, Choudary PV, Martin BM, et al. A mutation in the human glucocerebrosidase gene in neuronopathic Gaucher disease. $N$ Engl f Med 1987;316:570.

32 Levy H, Or A, Eyal N, et al. Molecular aspects of Gaucher disease. Dev Neurosci 1991;13:352-62.

33 Grace ME, Berg A, He G, Goldberg L, Horowitz M, Grabowski GA. Gaucher disease: heterologous expression of two alleles associated with neuronopathic phenotypes. Am 7 Hum Genet 1991;646-55.

34 Beutler E, Gelbart T, Kuhl W, Zimran A, West C. Mutations in Jewish patients with Gaucher disease. Blood 1992;79:1662-6.

35 Latham T, Grabowski GA, Theophilus BDM, Smith FI. Complex alleles of the acid $\beta$-glucosidase gene in Gaucher disease. Am 7 Hum Genet 1990;47:79-86.

36 Eyal N, Wilder S, Horowitz M. Prevalent and rare mutations in Gaucher patients. Gene 1990;96:277-63.

37 Zimran A, Sorge J, Gross A, Kubitz M, West C, Beutler E. A glucocerebrosidase fusion gene in Gaucher disease. Implications for the molecular anatomy, pathogenesis and diagnosis of this disorder. $\mathcal{F}$ Clin Invest 1990;85:219-22.

38 Firon N, Eyal N, Kolodny EH, Horowitz M. Genotype assignment in Gaucher disease by selective amplification of the active glucocerebrosidase gene. Am f Hum Genet 1990;46:527-32.

39 Widgerson $M$, Firon N, Horowitz Z, et al. Characterization of mutation in Gaucher patients by cDNA cloning. $A m \mathcal{F}$ Hum Genet 1989;44:365-77.

40 Beutler E, Gelbart T, West C. The facile detection of the nt 1226 mutation of glucocerebrosidase gene by "mismatched" PCR. Clin Chim Acta 1990;194:161-6.

41 Beutler E, Nguyen NJ, Henneberger MW, et al. Gaucher disease: gene frequencies in the Ashkenazi Jewish populadisease: gene frequencies in the Ashken
tion. Am $₹$ Hum Genet 1993;52:85-8.

42 Dahl N, Lagerström M, Erikson A, Pettersson U. Gaucher disease type III (Norrbottnian type) is caused by a single mutation in exon 10 of glucocerebrosidase gene. $A m \mathcal{F}$ mutation in exon 10 of gluco

43 Conzelman E, Sandhoff K. Biochemical basis of late-onset neurolipidosis. Dev Neurosci 1991;13:197-204.

44 Zimran A, Gross E, West C, Sorge J, Kubitz M, Beutler E. Prediction of severity of Gaucher by identification at DNA level. Lancet 1989;ii:349-52.

45 Sidranski E, Tsuji S, Martin BM, Stubblefield B, Ginns E. DNA mutation analysis of Gaucher patients. Am f Hum Genet 1992;42:331-6.

46 Masuno $M$, Tomatsu S, Sukegawa K, Orii T. Non-existence of tight association between Leu444Pro mutation and phenotypes of Gaucher patients: high frequency of Nci 1 polymorphism in the non-neuronopathic form. Hum Genet 1990;84:203-6.

47 Kawame H, Eto Y. A new glucocerebrosidase gene missense mutation responsible for neuronopathic Gaucher disease mutation responsible for neuronopathic Gaucher disease 48 Ohashi T, Hong CM, Weiler S, et al. Characterization of human glucocerebrosidase from different mutant alleles. $\mathcal{f}$ Biol Chem 1991;266:3661-9.

49 Van Weely S, van den Berg M, Barranger JS, Sa Miranda SC, Tager JM, Aerts JMFG. Role of $\mathrm{pH}$ in determining the cell type-specific residual activity of glucocerebrosidase in type 1 Gaucher disease. $\mathcal{f}$ Clin Invest 1993;91:1167-75.

50 Tybulwicz VLJ, Tremblay ML, La Marca ME, et al. Animal models of Gaucher's disease from targetted disruption of a mouse glucocerebrosidase gene. Nature 1992;357:407-11. 